\title{
Function of $\gamma \delta$ T cells in tumor immunology and their application to cancer therapy
}

\author{
Jang Hyun Park ${ }^{1}$ and Heung Kyu Lee (1)
}

\begin{abstract}
T cells of the $\gamma \delta$ lineage are unconventional T cells with functions not restricted to MHC-mediated antigen presentation. Because of their broad antigen specificity and NK-like cytotoxicity, $\gamma \delta$ T-cell importance in tumor immunology has been emphasized. However, some $\gamma \delta$ T-cell subsets, especially those expressing IL-17, are immunosuppressive or tumor-promoting cells. Their cytokine profile and cytotoxicity are seemingly determined by cross-talk with microenvironment components, not by the $\gamma \delta T C R$ chain. Furthermore, much about the TCR antigen of $\gamma \delta T$ cells remains unknown compared with the extreme diversity of their TCR chain pairs. Thus, the investigation and application of $\gamma \delta$ T cells have been relatively difficult. Nevertheless, $\gamma \delta$ T cells remain attractive targets for antitumor therapy because of their independence from MHC molecules. Because tumor cells have the ability to evade the immune system through $\mathrm{MHC}$ shedding, heterogeneous antigens, and low antigen spreading, MHC-independent $\gamma \delta$ $T$ cells represent good alternative targets for immunotherapy. Therefore, many approaches to using $\gamma \delta T$ cells for antitumor therapy have been attempted, including induction of endogenous $\gamma \delta T$ cell activation, adoptive transfer of expanded cells ex vivo, and utilization of chimeric antigen receptor (CAR)-T cells. Here, we discuss the function of $\gamma \delta$ $T$ cells in tumor immunology and their application to cancer therapy.
\end{abstract}

\section{Introduction}

The novel T-cell $\gamma \delta$ line was identified upon the discovery of the $\gamma$ gene in $1984^{1,2}$. Furthermore, $\gamma \delta \mathrm{T}$ cells express $\gamma \delta$ T-cell receptor $(\gamma \delta$ TCR) but not $\alpha \beta T C R . \gamma \delta$ $\mathrm{T}$ cells constitute part of the "unconventional" T-cell subset and function in unique roles, such as stress surveillance $^{3}$. The most unique characteristics of $\gamma \delta \mathrm{T}$ cells are migration to peripheral tissues rather than lymphoid organs and functions independently of major histocompatibility complex (MHC)-dependent antigen presentation ${ }^{4}$. In mice, $\gamma \delta \mathrm{T}$ cells first develop in the embryonic thymus. Compared with conventional $\mathrm{T}$ cells, which are derived from double-positive (DP) thymocytes, $\gamma \delta \mathrm{T}$ cells are derived from $\mathrm{CD} 4^{-} / \mathrm{CD}^{-}$double-negative (DN) thymocytes. From the DN stage, functionally distinct $\gamma \delta \mathrm{T}$ cells develop at different stages along with varying TCR pairs ${ }^{5}$. The anatomical localization of $\gamma \delta$

\footnotetext{
Correspondence: Heung Kyu Lee (heungkyu.lee@kaist.ac.kr)

${ }^{1}$ Graduate School of Medical Science and Engineering, Korea Advanced

Institute of Science and Technology (KAIST), Daejeon 34141, Republic of Korea
}

$\mathrm{T}$ cells is also different from each other. For example, ${\mathrm{V} \gamma 5^{+}}^{+}$dendritic epidermal $\mathrm{T}$ cells (DETCs; Tonegawa nomenclature) are located in the epidermis of the skin, whereas $\mathrm{V} \gamma^{+}{ }^{+}$cells reside in the meninges, genital tract, and lungs. $\mathrm{V} \gamma^{+}{ }^{+}$cells are located in the liver, lymphoid organs, and skin, whereas $\mathrm{V} \gamma^{+}$intraepithelial lympho-

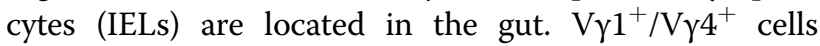
are generated during and after the postnatal period and are distributed systemically, similar to adaptive immune cells ${ }^{6}$. Because $\gamma \delta \mathrm{T}$-cell subsets are not conserved between mice and humans, the translation of results is difficult. For example, DETCs do not exist in human skin. Usually, $\gamma$ chains are used to classify murine $\gamma \delta \mathrm{T}$ cell subsets; however, $\delta$ chains are used to classify these sets in humans ${ }^{7}$. Furthermore, $\gamma \delta \mathrm{T}$ cells constitute a minor population in both mice and humans. However, they participate in host defense against a variety of conditions, including viral and bacterial infections and cancer ${ }^{8}$. Specifically, due to their strong cytotoxicity and unrestricted MHC features, $\gamma \delta \mathrm{T}$ cells are thought to be a good 
alternative therapeutic target for cancer ${ }^{7}$. In this review, we discuss the complex potential role of $\gamma \delta \mathrm{T}$ cells in the tumor microenvironment (TME) and the possibility of using $\gamma \delta \mathrm{T}$-cell-based antitumor immunotherapy in the future.

\section{The antitumor function of $\gamma \delta \mathrm{T}$ cells Direct cytotoxicity}

$\gamma \delta \mathrm{T}$ cells are frequently observed in multiple tumor tissues, and their presence is thought to be a favorable prognostic factor ${ }^{9}$. In addition, $\gamma \delta \mathrm{T}$ cells are known as stress sensors. Ligation between stress-induced molecules, such as MHC class I polypeptide-related sequence A (MICA) and natural killer group 2 member D (NKG2D), provokes target-specific killing ${ }^{10}$. Transformation is one cellular stress mechanism that induces the expression of NKG2D ligands (NKG2DLs) ${ }^{11}$. Thus, $\gamma \delta \mathrm{T}$ cells are generally considered cytotoxic and antitumor lymphocytes (Fig. 1). The Hayday group showed that $\gamma \delta \mathrm{T}$ cells can recognize and regulate cutaneous malignancy using PDV cell line injection and methylcholanthrene (MCA)- and dimethylbenz[a]anthracene (DMBA)-induced cutaneous tumors $^{12}$. The antitumor function of $\gamma \delta \mathrm{T}$ cells has been extended to other tumors, such as B-cell lymphoma, prostate cancer, melanoma, and mesenchymal glioblastoma $^{13-16}$. In addition to NKG2D, $\gamma \delta \mathrm{T}$ cells need various types of receptors depending on the context. For

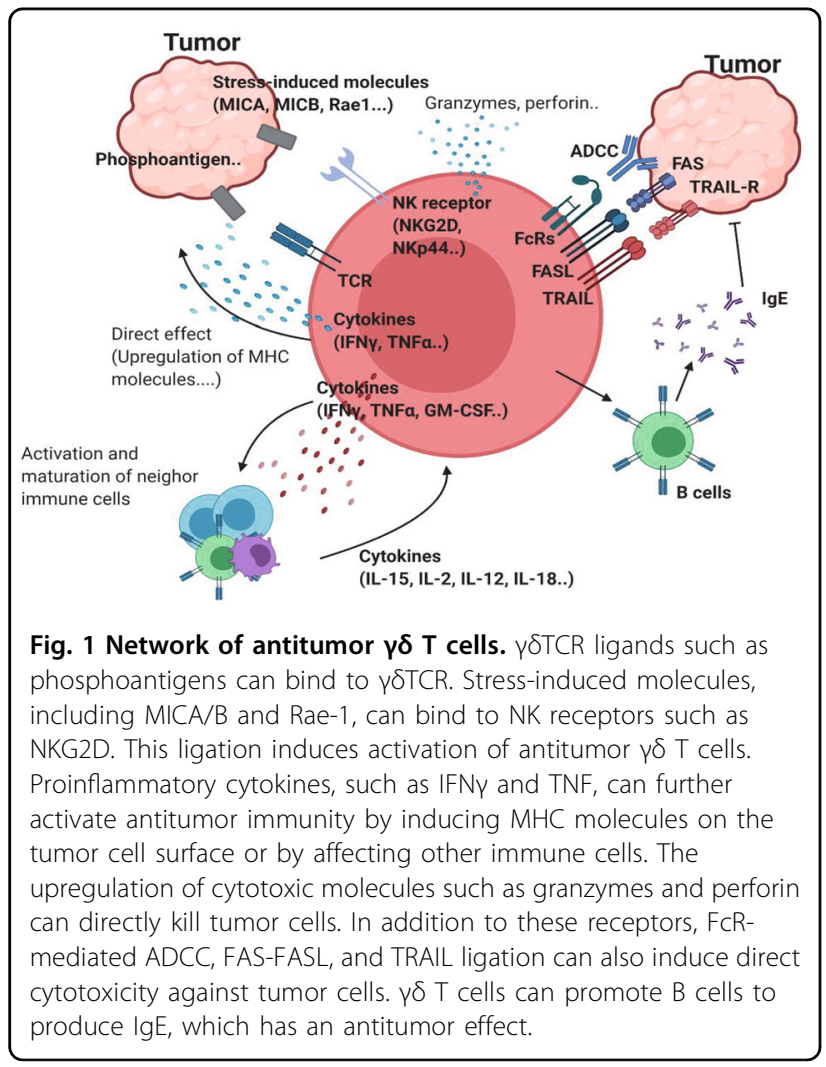

example, $\gamma \delta$ T cells need both $\gamma \delta \mathrm{TCR}$ and NKG2D to kill TCCSUP human transitional cell carcinoma cells ${ }^{17}$. However, $\gamma \delta \mathrm{T}$ cells need only $\gamma \delta \mathrm{TCR}$ to target the zoledronate-treated human rhabdomyosarcoma (RMS) cell line ${ }^{18}$. Daudi cells, which express endogenous $\gamma \delta$ TCR ligands but not MHC class I or MICA ligands, are not dependent on NKG2D. Furthermore, the RMA murine lymphoma cell line does not express NKG2DL ${ }^{19}$. Other NK receptors (NKRs), including CD226 (DNAM-1), natural cytotoxicity-triggering receptor 3 (NCR3; NKp30), and NCR2 (NKp44), also participate in tumor recognition $^{20}$. TNF receptors, such as TNF-related apoptosisinducing ligand (TRAIL) and Fas ligand (FASL), can also kill tumor cells ${ }^{7}$. Human $\gamma \delta \mathrm{T}$ cells express CD16 and participate in inducing antibody-dependent cellular cytotoxicity $^{21}$.

\section{Interaction between the microenvironment and $\gamma \delta \mathrm{T}$ cells}

Certain environments regulate $\gamma \delta \mathrm{T}$ cells for optimal activation. For example, IL-2 and IL-15 are the main inducers of cytotoxic $\gamma \delta$ T cells ${ }^{22}$. IL-12, IL-18, IL-21, and IL-36y are also involved in IFNY production and cytotoxicity $^{7}$. In contrast, IL-10 and transforming growth factor-beta (TGF $\beta$ ) secreted from regulatory $\mathrm{T}$ cells or myeloid cells can negatively regulate $\gamma \delta \mathrm{T}$ cells ${ }^{23}$. Although $\gamma \delta \mathrm{T}$ cells are known to function independent of MHC recognition, MHC-restricted $\gamma \delta$ TCRs in MART1and gp100-reactive $\gamma \delta \mathrm{T}$ cells have been reported ${ }^{24}$. Environmental factors also affect $\gamma \delta$ T-cell function. For example, reactive oxygen species, oxygen tension, and cholesterol can influence IFN $\gamma$ and/or NKR expres$\operatorname{sion}^{25-27}$. In the case of high-grade glioma, tumorinfiltrated $\gamma \delta \mathrm{T}$ cells have a high apoptosis rate. The number of $\gamma \delta \mathrm{T}$ cells decreases dramatically at the terminal stage of the disease ${ }^{28}$. These results suggest that the TME attenuates $\gamma \delta$ T-cell responses in multiple ways. Because $\gamma \delta$ T cells are actively recruited to the TME and have the potential to kill target cells in vitro, our lack of understanding of how the TME suppresses $\gamma \delta \mathrm{T}$ cells remains a major challenge.

\section{$\gamma \delta \mathrm{T}$ cells bridge innate and adaptive antitumor responses}

Because $\gamma \delta \mathrm{T}$ cells are early stress responders and possess adaptive immunity-like characteristics, they are thought to create a link between innate and adaptive immune responses. $\gamma \delta \mathrm{T}$ cells comprise various subsets categorized by their combination of TCR chains. SRY-box transcription factor $(\text { Sox } 13)^{+}$fetal progenitors for $\mathrm{V} \gamma 2^{+}$ $\mathrm{T}$ cells originate independently of $\gamma \delta \mathrm{TCR}^{29}$. Subsets of innate-like $\gamma \delta$ T cells do not require TCR engagement but might be dependent on cytokines or innate receptors, such as NKG2D. However, $\gamma \delta$ T cells usually need a TCR signal for their activation and development. Studies have demonstrated the effect of different antigens on $\gamma \delta$ T-cell 


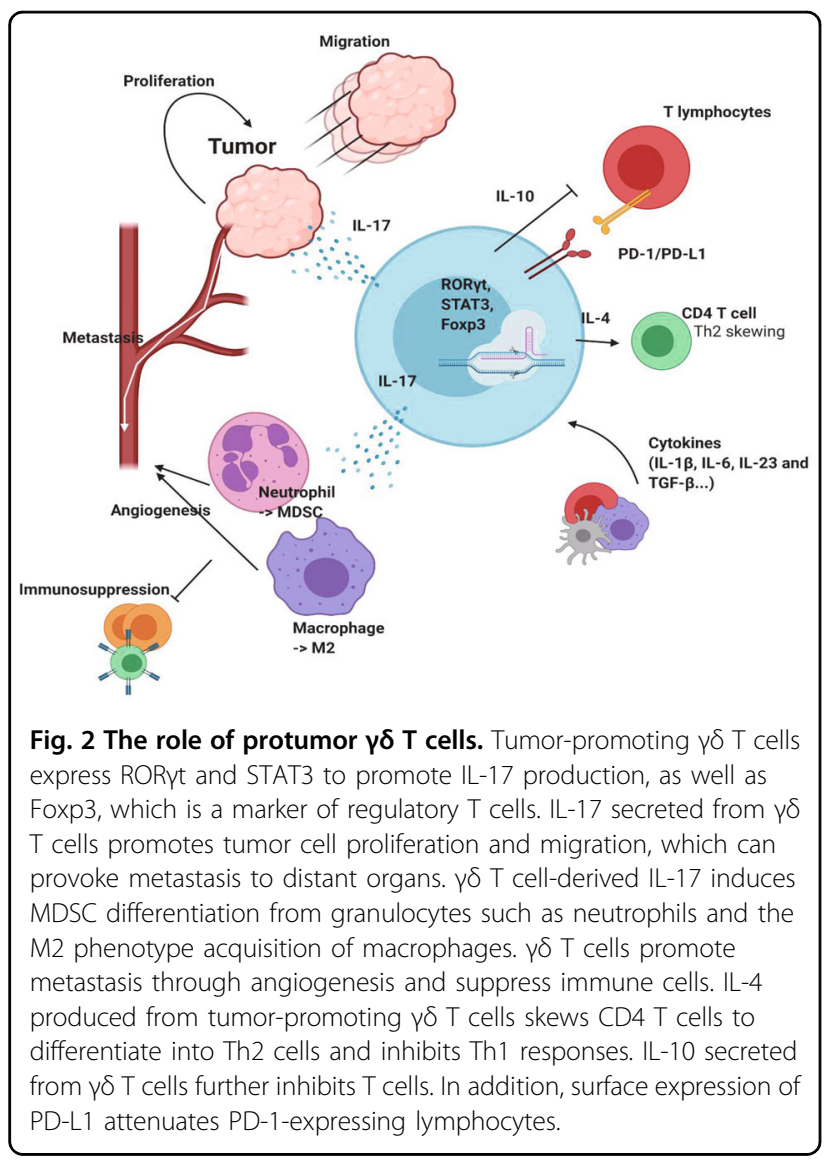

development and activation ${ }^{30,31}$. Furthermore, persistent expansion of $\gamma \delta \mathrm{T}$ cells in cytomegalovirus (CMV)infected patients following kidney transplantation has been observed ${ }^{32}$. Proinflammatory $\alpha \beta$ - and $\gamma \delta$ TCR-coexpressing $\mathrm{T}$ cells have also been reported ${ }^{33}$, suggesting that subsets of $\gamma \delta \mathrm{T}$ cells possess adaptive, not innate, features. Research using tuberculosis infection in nonhuman primates has shown that $\mathrm{V} \gamma 9 \mathrm{~V} \delta 2 \mathrm{~T}$ cells can induce memory responses ${ }^{34}$. Thus, $\gamma \delta \mathrm{T}$ cells have both innate and adaptive functions and can link both responses ${ }^{6}$. In cases of cancer, it is still unclear whether $\gamma \delta \mathrm{T}$ cells act more innately or adaptively. However, we hypothesize that innate-like $\gamma \delta \mathrm{T}$ cells may acutely respond to tumor cells via stress sensing, whereas adaptive $\gamma \delta \mathrm{T}$ cells may establish durable antitumor responses in an antigenspecific manner. Because $\gamma \delta \mathrm{T}$ cells have been shown to expand following CMV infection, CMV-positive tumor cells may be good models for investigating adaptive $\gamma \delta$ $\mathrm{T}$ cells. CMV-specific $\mathrm{T}$ cells are reactive to glioblastoma multiforme (GBM) cells ${ }^{35}$. Thus, although controversial, cell therapy using $\gamma \delta \mathrm{T}$ cells against CMV-positive tumors may be applicable. On the other hand, $\gamma \delta \mathrm{T}$ cells are an early source of IFN $\gamma$ in the TME ${ }^{36}$. IFNY derived from $\gamma \delta$ $\mathrm{T}$ cells can amplify the production of $\alpha \beta \mathrm{T}$ cells and induce the expression of MHC class I molecules on tumor cells ${ }^{37}$. In addition, antigen presentation and costimulation of $\alpha \beta \mathrm{T}$ cells derived from $\gamma \delta \mathrm{T}$ cells have been observed in gastric cancer ${ }^{38}$. The antigen-specific T-cell expansion has been successfully induced by coculture with $\gamma \delta \mathrm{T}$ cells $^{39}$. In addition to CD4 T cells, $\gamma \delta \mathrm{T}$ cells can also boost $\mathrm{B}$ cells. Topical 12-dimethylbenz[a] anthracene (DMBA)-induced tumorigenesis promoted B-cell IgE production in a $\mathrm{V} 5^{+} \mathrm{T}$ cell-dependent manner. IgE was also shown to protect a host from carcinogenesis ${ }^{40}$. GM-CSF produced by $\gamma \delta \mathrm{T}$ cells controlled $\mathrm{CD}_{103^{+}}$dendritic cells (DCs) ${ }^{41}$. These findings show that $\gamma \delta \mathrm{T}$ cells can communicate with multiple immune cells surrounding the TME. In summary, although $\gamma \delta$ $\mathrm{T}$ cells are considered innate immune cells, subsets of $\gamma \delta$ $\mathrm{T}$ cells exhibit adaptive characteristics and can serve as a bridge between innate and adaptive immune responses.

\section{The protumor function of $\gamma \delta$ T cells}

\section{The protumor function of IL-17-producing $\gamma \delta \mathrm{T}$ cells}

In general, IL-17A-producing $\gamma \delta \mathrm{T}$ cells are considered to be tumor-promoting cells (Fig. 2). IL-17-producing $\gamma \delta$ $\mathrm{T}$ cells are rarely found in healthy humans ${ }^{42}$. However, in multiple tumor models, tumor injection induces IL-17A production by $\gamma \delta \mathrm{T}$ cells. Furthermore, IL-17-deficient animals show reduced tumor mass in breast cancer, hepatocellular carcinoma, lung cancer, and melanoma ${ }^{7}$. The tumor-promoting function of IL-17A is mainly manifested by angiogenesis and metastasis. In fibrosarcoma, circulating $\gamma \delta \mathrm{T}$ cells, but not $\mathrm{V} \gamma 5^{+}$cells, produce IL-17A and promote angiogenesis ${ }^{43}$. In mice, IL-17 is usually produced by $\mathrm{V} 4^{+}$or $\mathrm{V} \gamma^{+}{ }^{+}$cells ${ }^{44}$. In humans, although IL-17 can be secreted by $\mathrm{V} \gamma 9 \mathrm{~V} \delta 2^{+} \mathrm{T}$ cells upon stimulation with antigens and cytokines, such as IL-1 $\beta$, IL-6, IL-23, and TGF $\beta^{45}$, IL-17 has been shown to be preferentially produced by $\mathrm{V} \delta 1^{+} \mathrm{T}$ cells ${ }^{46}$. Because tissueresident innate-like $\gamma \delta \mathrm{T}$ cells are more prone to producing IL-17 than circulating $\gamma \delta$ T cells, which preferentially produce IFN $\gamma$, tissue-resident $\mathrm{V} \delta 1^{+} \mathrm{T}$ cells, not $\mathrm{V} \delta 2^{+}$ $\mathrm{T}$ cells, may be main sources of IL-17. However, biology seems is complicated. The cytokine profile of $\gamma \delta \mathrm{T}$ cell subsets is highly dependent on context. In breast cancer, tissue-resident $\mathrm{V} \delta 1^{+} \mathrm{T}$ cells are skewed toward cytolysis and IFNY production but not IL-17 production ${ }^{47}$. Thus, the determination of whether $\gamma \delta$ T cells produce IL-17 or IFN $\gamma$ based on TCR chains might be meaningless. IL-17 contributes to tumor progression in multiple ways. As mentioned above, IL-17 can promote angiogenesis through direct signaling on endothelial cells ${ }^{43}$. However, IL-17 can promote angiogenesis indirectly. IL-17 can promote macrophages to make angiogenic factors such as vascular endothelial growth factor ${ }^{48}$. Furthermore, IL-17 can induce M2 macrophage polarization ${ }^{49}$. In addition, $\gamma \delta$ $\mathrm{T}$ cells can recruit neutrophils and facilitate their expansion in the TME through IL-17 and G-CSF ${ }^{50}$. On the 
other hand, through the PI3K/AKT signaling pathway, IL-17 can directly activate tumor cells to be more migratory $^{51}$. Despite these findings, the roles of IL-17 remain unclear, depending on the tumor model and specimen. For example, two studies focusing on colon cancer have shown opposite conclusions. One study suggested that high numbers of $\gamma \delta \mathrm{T}$ cells reside in the TME and act as the main sources of IL- $17^{52}$. However, the results from another study suggest that $\gamma \delta \mathrm{T}$ cells are major sources of IFN $\gamma$, not IL-17, and constitute a very minor population ${ }^{53}$. Studies focusing on brain tumors are present contradictory findings. IL-17A can promote the migration of U87 MG and U251 human GBM cells ${ }^{51}$, and inhibition of IL-17A can extend the overall survival of patient-derived tumor-bearing immunodeficient mice ${ }^{54}$. However, one study showed that GBM patients who express high levels of IL-17 survive longer than those expressing lower levels ${ }^{55}$. These data suggest that the role of IL-17 varies depending on the context and network of cells in the TME.

\section{Other mechanisms of tumor-promoting $\gamma \delta$ T cells}

In addition to IL-17, other mediators secreted from $\gamma \delta$ $\mathrm{T}$ cells can promote tumor progression. $\mathrm{CD} 39^{+} \gamma \delta \mathrm{T}$ cells can suppress immune responses through the adenosine pathway and recruit myeloid-derived suppressor cells in colorectal cancer ${ }^{56}$. These $\mathrm{CD} 39^{+} \gamma \delta \mathrm{T}$ cells express FOXP3, a marker of regulatory T cells. TGF $\beta$ treatment increases FOXP3 expression in human peripheral blood mononuclear cell (PBMC)-derived $\gamma \delta$ T cells. FOXP3 ${ }^{+} \gamma \delta$ $\mathrm{T}$ cells can inhibit the proliferation of $\mathrm{T}$ cells derived from $\mathrm{PBMCs}^{57}$. In a murine sarcoma model, $\gamma \delta \mathrm{T}$ cells secrete galectin-1, which suppresses cytotoxic CD8 $\mathrm{T}$ cells ${ }^{58}$. In murine pancreatic cancer, $\gamma \delta$ T cells express programmed death-ligand 1 (PD-L1) and galectin-9 to suppress cytotoxic $\mathrm{T}$ cells ${ }^{59}$. IL-4-conditioned $\gamma \delta \mathrm{T}$ cells are more likely to form a subset of $\mathrm{V} \delta 1^{+} \mathrm{T}$ cells that inhibit T-cell proliferation in an IL-10-dependent manner ${ }^{60}$.

\section{Regulation of $\gamma \delta \mathrm{T}$ cells}

\section{Recruitment of $\gamma \delta \mathrm{T}$ cells}

CC-chemokine receptor 6 (CCR6) is a well-defined chemokine receptor of IL-17-producing $\gamma \delta \mathrm{T}$ cells. $\mathrm{V}_{4} 4^{+}$ and $\mathrm{V} \gamma_{\gamma} 6^{+} \mathrm{T}$-cell localization to the skin is dependent on CCR6. However, activation of IL-17-producing $\gamma \delta \mathrm{T}$ cells leads to the downregulation of CCR6 in an IL-1 $\beta-$, IL-23-, and/or IL-7-manner. Because IL-17-producing $\gamma \delta \mathrm{T}$ cells coexpress CCR2, CCR6 downregulation promotes CCR2 dependency. The migration of $\gamma \delta \mathrm{T}$ cells into inflammatory sites has been shown to be dependent on CCR2 ${ }^{61}$. Furthermore, IFN $\gamma$-producing $\gamma \delta \mathrm{T}$ cells also express CCR2. However, blood-derived $\mathrm{V} \delta 1^{+} \mathrm{T}$ cells, but not $\mathrm{V} \delta 2^{+} \mathrm{T}$ cells, express CCR $2^{62}$. V $\delta 1^{+} \mathrm{T}$ cells also express CXCR3; however, $\mathrm{V} \delta 2^{+} \mathrm{T}$ cells distinctly express
CCR ${ }^{63,64}$. In the TME, $\gamma \delta \mathrm{T}$ cells seem to be recruited mainly by CCR2 and CXCR3 ${ }^{65}$. However, an accurate characterization of chemokine receptor expression on $\gamma \delta$ $\mathrm{T}$ cells remains to be performed. Adhesion molecules, including LFA-1, are also important for $\gamma \delta$ T-cell recruitment ${ }^{66}$.

\section{T-cell receptor signaling}

The $\gamma \delta$ TCR complex is composed of $\gamma \delta T C R$ and other CD3 chains. TCR signal strength is important for $\gamma \delta \mathrm{T}$ cell development and activation (Fig. 3a). A strong TCR signal through $\gamma \delta$ TCR causes $\alpha \beta / \gamma \delta$ common precursors to differentiate into $\gamma \delta \mathrm{T}$ cells ${ }^{67}$. In general, ligated TCR complexes induce the phosphorylation of immunoreceptor tyrosine-based activation motifs (ITAMs) by SRC family kinases (SFKs), lymphocyte-specific protein tyrosine kinases (LCKs), and FYN proto-oncogenes (FYNs). However, although in contrast to other $\mathrm{T}$ cells, $\gamma \delta \mathrm{T}$ cells do not express CD4 or CD8; therefore, the mechanism by which TCR signaling is mediated remains unclear. One possible explanation involves extracellular signal-regulated kinase (ERK) phosphorylation, which can activate SFKs ${ }^{68}$. The requirement for TCR signaling is dependent on context (cytokines, inflammation, etc.) or cellular subsets defined by their $\gamma$ and $\delta$ chains. IFN $\gamma$-producing $\gamma \delta$ T cells, but not IL-17producing $\gamma \delta \mathrm{T}$ cells, require the thymic expression of T10/T22 $2^{30}$. Thymic selection and maintenance of intraepithelial T-cell protein 1 (Skint1) also lead to IFN $\gamma$-producing DETCs, not to IL-17 production, because it suppresses SOX13 and RORyt ${ }^{31}$. Sox13expressing DN1d thymocytes, which are progenitors of IL-17-producing $\gamma \delta \mathrm{T}$ cells, do not require TCR expression or signaling. However, phycoerythrin (PE)specific $\gamma \delta$ T cells were shown to secrete IL-17 in a TCRdependent manner. In addition, signaling through the zeta chain of $\mathrm{T}$ cell receptor-associated protein 70 (ZAP70) is required for the development of IL-17producing $\gamma \delta \mathrm{T}$ cells but not IFN $\gamma$-producing $\gamma \delta$ $\mathrm{T}$ cells ${ }^{69}$. In summary, the requirement for TCR signaling is dependent on subsets, context, and antigens.

Recent interesting research has suggested that abrogating $\gamma \delta$ TCR rearrangements lead to innate lymphoid cell 2 (ILC2) generation. TCR $\delta$-deficient mice showed an increase in ILC2s. Thus, we need to be careful when interpreting phenotypes using TCR $\delta$-deficient mice $^{70}$. Because the identity of general antigens for $\gamma \delta \mathrm{T}$ cells remains unclear, knowledge about specific tumor antigens is even nebulous. Annexin A2, which can bind to the V83 TCR, is expressed in multiple cancer cell types, including endometrial, breast, and glioblastoma cells ${ }^{71}$. p-Ag-bound butyrophilin 3A (BTN3A) isoforms that can activate $\mathrm{V} \gamma 9 \mathrm{~V} \delta 2 \mathrm{~T}$ cells through TCR signaling to play critical roles in regulating antitumor immunity ${ }^{72}$. 


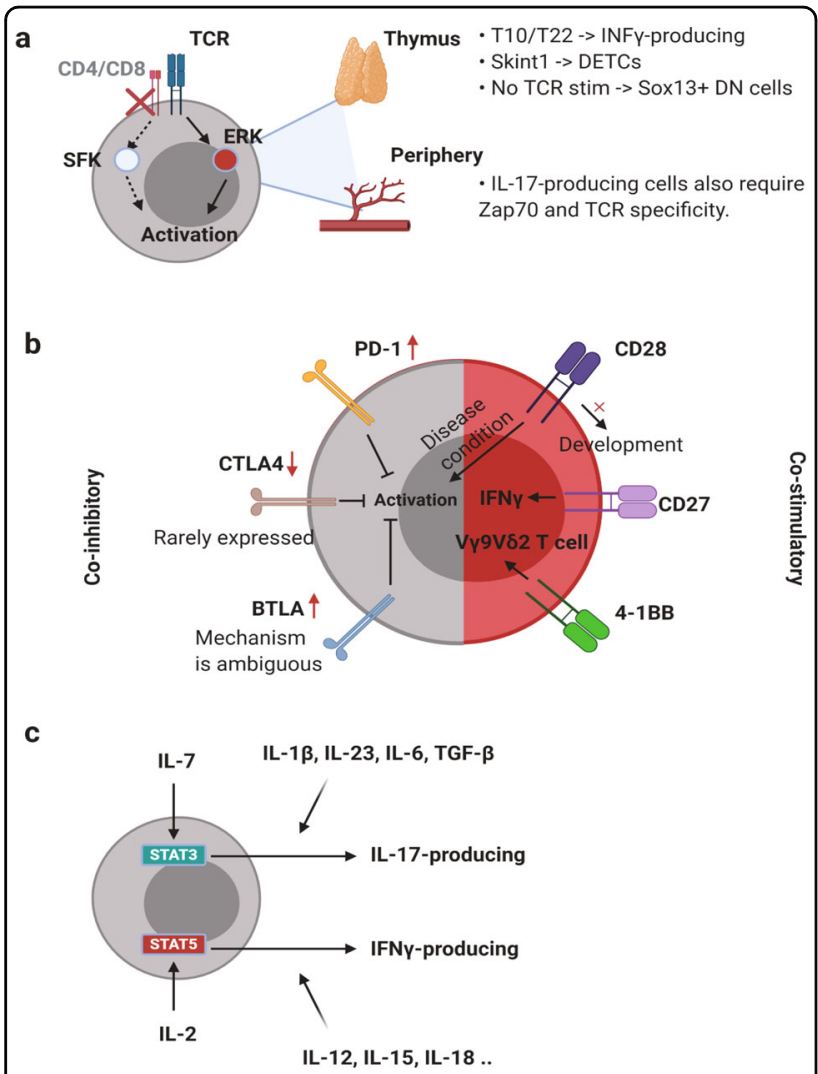

Fig. 3 Regulation of $\gamma \delta$ T cells. a Although there are exceptions, $\gamma \delta$ $T$ cells generally do not express CD4 or CD8. Therefore, SFK-mediated activation cannot be used to activate $\gamma \delta$ T cells. However, the ERK pathway is activated. For the normal development of $\gamma \delta$ T cells, TCR signaling is important. T10/T22 and Skint1 are well-defined antigens for $\gamma \delta T C R . T 10 / T 22-$ mediated signaling induces IFNy-producing $\gamma \delta T$ cells, whereas the Skint1 signaling induces DETC development. Some innate subsets of $\gamma \delta$ T cells do not require TCR stimulation during

development. Although several reports have demonstrated that innate IL-17-producing $\gamma \delta$ T cells do not require TCR signaling, other studies have shown that TCR specificity or Zap70 phosphorylation is needed for full $\gamma \delta T$ cell activation. b Coinhibitory molecules, including PD-1, CTLA4, and BTLA, can suppress $\gamma \delta$ T cells. PD-1 and BTLA are frequently expressed by $\gamma \delta T$ cells. However, CTLA4 is rarely expressed on the $\gamma \delta T$ cell surface. Costimulatory molecules such as CD28, CD27, and 4-1BB are needed for development or activation. CD28 is needed for

advancing disease conditions but not its development. However, CD27 is needed for the development and proper expression of IFN- - . 4-1BB is known to enhance Vy9V82 T cells. However, the roles and mechanisms of these and other coinhibitory/stimulatory molecules remain unclear. c Various cytokines induce $\gamma \delta T$ cell differentiation, leading to different functions. The $\gamma \delta$ T-cell differentiation dichotomy is mainly affected by cytokine signals.

Various kinds of ligands of $\gamma \delta \mathrm{TCR}$ (Annexin A2, tRNA synthetases, T10/T22, Skint-1, etc.) are currently being discovered, and they are expressed by multiple cancer cell types ${ }^{73}$. The identification of antigens is difficult because of the relatively low affinity of TCRs. If we can identify and categorize ligands similar as we did with vitamin B-MAIT cells, lipid antigen-NKT cells, and peptide antigen- $\alpha \beta \mathrm{T}$ cells, our understanding of $\gamma \delta$ T-cell involvement in antitumor immunity will be greatly enhanced.

\section{Costimulatory and inhibitory molecules}

Costimulatory molecules prevent anergy and enhance Tcell activation (Fig. 3b). CD28, the most well-understood costimulatory molecule, is expressed on $\gamma \delta \mathrm{T}$ cells and promotes proliferation and survival through IL-2 signaling $^{74}$. However, the requirement for CD28 signaling differs depending on the disease stage and/or tumor type $\mathrm{fu}^{74,75}$. Thus, the requirement should be determined accordingly. CD28 is not necessary for the development of $\gamma \delta \mathrm{T}$ cells. However, CD27 is important for IFN $\gamma$-producing $\gamma \delta$ $\mathrm{T}$ cells ${ }^{76}$. CD27 is often used as a marker for IFNy-producing $\gamma \delta$ T cells because CD27-dependent division of $\gamma \delta$ $\mathrm{T}$ cells occurs in the thymus. However, its role in the TME remains unclear. Because the CD70-CD27 interaction can enhance IFN $\gamma$ production and the survival of $\mathrm{V} \gamma 9 \mathrm{~V} \delta 2$ $\mathrm{T}$ cells in vitro ${ }^{77}$, it might be an applicable target in antitumor $\gamma \delta$ T-cell therapy. CD137 (4-1BB) can enhance $\mathrm{V} \gamma 9 \mathrm{~V} \delta 2 \mathrm{~T}$-cell function following influenza or Listeria infection. Thus, CD137 may also be involved in the antitumor function of $\gamma \delta \mathrm{T}$ cells ${ }^{78,79}$.

The reinvigoration of exhausted or inhibited $\mathrm{T}$ cells is an increasingly studied approach to immunotherapy. Because $\gamma \delta \mathrm{T}$ cells play crucial roles in various types of tumors, understanding the inhibitory signal of $\gamma \delta$ T cells might be beneficial for developing effective immunotherapies. Although $\gamma \delta \mathrm{T}$ cells rarely express cytotoxic T-lymphocyte-associated protein-4 (CTLA-4), upon activation, $\gamma \delta \mathrm{T}$ cells rapidly upregulate programmed cell death protein-1 (PD-1) and B- and T-lymphocyte attenuator (BTLA) ${ }^{80}$. Although PD-1 signaling exerts an inhibitory effect on IFN $\gamma$ production, phospho-antigenmediated activation of $\mathrm{V} \gamma 9 \mathrm{~V} \delta 2 \mathrm{~T}$ cells can overcome PD1 signaling ${ }^{81}$. Other costimulatory molecules, such as OX40 and CD40L, and coinhibitory molecules, including $\mathrm{V}$-domain immunoglobulin suppressor of $\mathrm{T}$ cell activation (VISTA), T-cell immunoreceptor with Ig and ITIM domains, or glucocorticoid-induced TNFR-related protein, might be involved in $\gamma \delta \mathrm{T}$-cell activation in various ways. Thus, a comprehensive investigation of these molecules is needed to develop effective $\gamma \delta \mathrm{T}$ cell-based therapies.

\section{Cytokines}

Cytokines such as IL-2 and IL-7 are essential for the survival and proliferation of $\mathrm{T}$ cells (Fig. 3c). Additional cytokines, including IL-4 and IL-12, are important for determining the differentiation fate of T cells. IL-7 and IL15 have been established as the most important cytokines for murine $\gamma \delta$ T-cell development and homeostasis. 


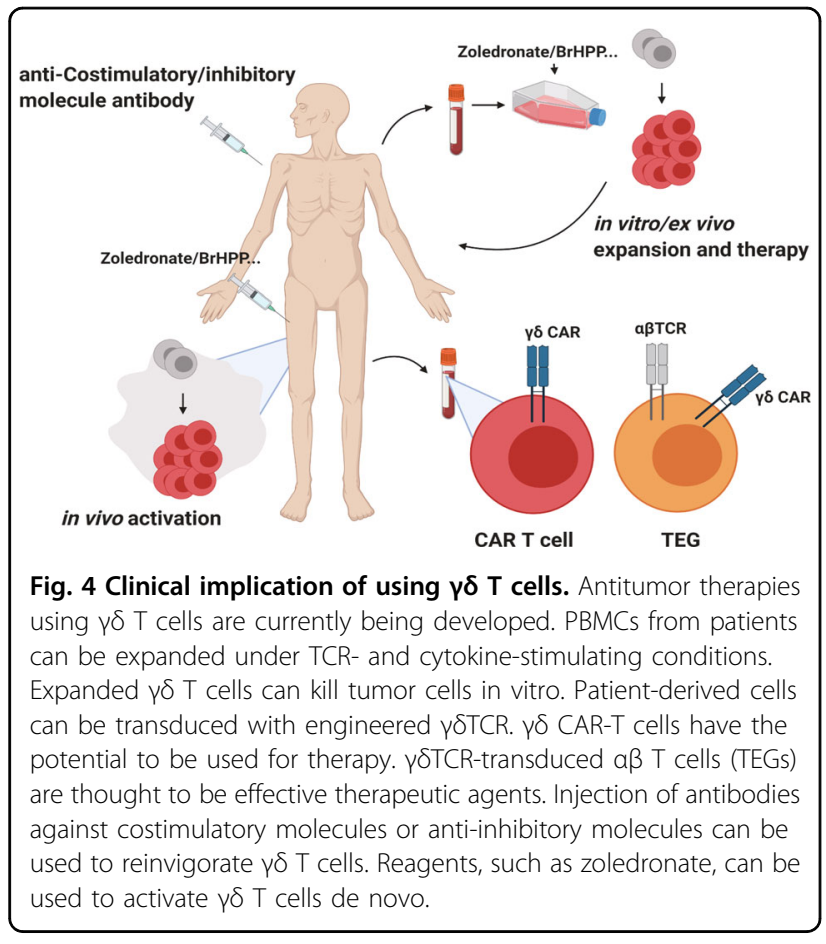

Conditional depletion of IL-7 from thymic epithelial cells resulted in a reduction in $\gamma \delta \mathrm{T}$ cells in multiple organs, such as the thymus, gut, and $\operatorname{skin}^{82}$. IL-7 is seemingly important for the early development of $\gamma \delta \mathrm{T}$ cells, especially IL-17-producing $\gamma \delta \mathrm{T}$ cells. IL-7 facilitates the selective expansion of IL-17-producing $\gamma \delta \mathrm{T}$ cells through signal transducer and transcription 3 (STAT3)-dependent signaling. This signaling pathway induces $\gamma \delta$ T-cell resistance to activation-induced cell death and prolifera$\operatorname{tion}^{83}$. However, gut-residing intraepithelial $\gamma \delta \mathrm{T}$ cells tend to be relatively more dependent on IL- $15^{84}$. On the other hand, IFN $\gamma$-producing $\gamma \delta \mathrm{T}$ cells require IL-15 and IL-2, but not IL- $7^{22}$. In addition to phospho-antigendependent TCR activation, IL- 2 and IL- 15 activate $\gamma \delta$ Tcell differentiation even though TCR signaling is dispensable for ERK activation and the expression of T-bet and Eomes ${ }^{22}$. Thus, IL-2 and IL-15 might be the most important cytokines in $\gamma \delta \mathrm{T}$ cell-based antitumor immunotherapy. In addition, IL-18 and IL-12 are involved in IFN $\gamma$ production in $\gamma \delta \mathrm{T}$ cells ${ }^{85,86}$, while IL- $1 \beta$ and IL23 promote IL-17 production $^{42,87}$.

\section{Clinical implications}

\section{Application of $\gamma \delta \mathrm{T}$ cells as prognostic factors}

Bisection of IL-17 and IFN $\gamma$ levels in $\gamma \delta$ T cells can be used for prognostics. IL-17-producing $\gamma \delta \mathrm{T}$ cells are associated with negative survival outcomes in patients with gallbladder or colon cancer ${ }^{52,88}$. Research has demonstrated that these cells are related not only to survival but also to tumor size, invasion, and metastasis.
In contrast, IFN $\gamma$-producing $\gamma \delta \mathrm{T}$ cells tend to be associated with a positive prognosis and prolonged survival ${ }^{88}$. Although one study suggested that $\gamma \delta$ T cells are mostly associated with a positive prognosis, this association remains ambiguous because discriminating the effects of $\gamma \delta \mathrm{T}$ cells from other lymphocytes in bioinformatics data is difficult ${ }^{9}$. Because IL-17 and IFN $\gamma$ can be produced by other cells, such as CD4/CD8-positive T cells and NK cells, further investigation is required to determine whether the presence of $\gamma \delta \mathrm{T}$ cells is a better prognostic factor than total cytokine production. Although recent highthroughput methods, such as single-cell RNA sequencing (scRNA-seq), cellular indexing of transcriptomes and epitopes by sequencing (CITE-seq), and spatial transcriptomics, are expensive and labor-intensive, using these methods to diagnose patients ultimately provides more insightful information.

\section{Current strategies for using $\gamma \delta$ T cells in the clinic}

V $\gamma 9 \mathrm{~V} \delta 2 \mathrm{~T}$ cells are common targets for $\gamma \delta \mathrm{T}$ cell-based immunotherapies (Fig. 4). These cells are a dominant type of blood-derived $\gamma \delta \mathrm{T}$ cells, and they are relatively easier to expand in vitro than $\mathrm{V} \delta 1^{+} \mathrm{T}$ cells ${ }^{7}$. One traditional strategy for using $\gamma \delta \mathrm{T}$ cells is adoptive cell therapy. Stimulation of $\gamma \delta \mathrm{T}$ cells using aminobisphosphonates or synthetic phosphoantigen analogs can induce $\mathrm{V} \gamma 9 \mathrm{~V} \delta 2 \mathrm{~T}$ cell expansion in vitro and ex vivo. Aminobisphosphonates, such as pamidronate and zoledronate, act as ligands for $\gamma \delta$ TCR to upregulate the mevalonate pathway and induce the production of pyrophosphate intermediates in cancer and myeloid cells. Synthetic phosphoantigen analogs, including bromohydrin pyrophosphate (BrHPP) and 2-methyl-3-butenyl-1-pyrophosphate (2M3B1PP), also directly act as ligands for $\gamma \delta \mathrm{TCR}^{89}$. Adoptive transfer of expanded $\mathrm{V} \gamma 9 \mathrm{~V} \delta 2 \mathrm{~T}$ cells was shown to be safe; however, the results from these studies have been disappointing. Several reasons for these failures have been suggested. First, the $V \gamma 9 V \delta 2$ TCR repertoire is too polyclonal to recognize tumors. Because recent studies have identified a novel $\gamma \delta \mathrm{TCR}$ ligand, selecting case-matched ligands for expansion is needed. For example, brain tumor cells express Annexin-A2, which can be targeted by $\gamma \delta \mathrm{T}$ cells ${ }^{90}$. However, additional studies are needed to examine whether Annexin A2stimulated $\gamma \delta \mathrm{T}$ cells are more efficient than traditionaldrug-stimulated $\gamma \delta$ T cells. Second, the TME can induce $\gamma \delta \mathrm{T}$ cells to dysfunctional and exhaustion. Oxygen tension and metabolic state can independently affect $\gamma \delta \mathrm{T}$ cell function. GBM-infiltrating $\gamma \delta \mathrm{T}$ cells are prone to becoming more apoptotic and dysfunctional as tumors progress $^{28}$. Thus, deletion of endogenous $\gamma \delta \mathrm{T}$ cells did not affect the overall survival of GBM-bearing mice. Furthermore, although the adoptive transfer of $\gamma \delta$ T cells ex vivo expanded extended the survival of human 
GBM-bearing immunodeficient mice, treatment with expanded murine $\gamma \delta \mathrm{T}$ cells was not beneficial for syngeneic GBM-bearing immunocompetent B6 mice ${ }^{28}$. This result suggests that $\gamma \delta \mathrm{T}$ cells are dramatically immunosuppressed in the TME. One study showed that hypoxia upregulates the cytotoxicity of $\gamma \delta \mathrm{T}$ cells in vitro. However, the survival of $\gamma \delta \mathrm{T}$ cells is downregulated by hypoxia $^{91}$. Another study showed that hypoxia inhibits tumor-derived exosome-mediated $\gamma \delta$ T-cell activation ${ }^{26}$. However, physiological normoxia (6\% oxygen) is hypoxic compared to the $20-21 \%$ oxygen levels of the in vitro environment used in this study; therefore, using a more physiologically relevant model system is recommended. Additional studies to understand which $\gamma \delta \mathrm{T}$ cells are regulated in the TME in vivo are required to develop more effective immunotherapies. Other drugs or therapies can affect $\gamma \delta$ T-cell immunity. For example, chemotherapy and radiotherapy not only kill tumor cells but also render $\gamma \delta \mathrm{T}$ cells fragile ${ }^{92}$. 5-Fluorouracil, doxorubicin, and cisplatin increase tumor cells' sensitivity to $\gamma \delta$ $\mathrm{T}_{\text {cells }}{ }^{93}$. The DNA methylation inhibitor decitabine was reported to upregulate the NKG2D ligand on tumor cells $^{94}$. Thus, to avoid unexpected side effects, the administration of drug combinations should be considered carefully. On the other hand, an adequate combination of conventional drugs may have antitumor effects mediated by $\gamma \delta \mathrm{T}$ cells. Reinvigorating $\gamma \delta \mathrm{T}$ cells by targeting coinhibitory/stimulatory molecules might be a good strategy. Anti-CTLA4 antibody treatment increased the frequency of ${\mathrm{V} \delta 2^{+}}^{\mathrm{T}}$ cells in melanoma patients ${ }^{95}$. However, combination therapy using anti-PD-1 and antiCTLA4 antibodies produced almost no change in $\gamma \delta \mathrm{T}$ cell levels ${ }^{96}$. Finding a reasonable target for reinvigorating $\gamma \delta \mathrm{T}$ cells might be more important before clinical use. Nanobodies or bispecific antibodies have also been used to increase the specificity and activity of $\gamma \delta \mathrm{T}$ cells ${ }^{7}$.

$\mathrm{V} \delta 1^{+} \mathrm{T}$ cells have been infrequently leveraged as therapeutic targets because validated agonizts for the $V \delta 1$ TCR have yet to be identified. However, a recent study showed that peripheral blood-derived cells can be expanded into ${\mathrm{V} \delta 1^{+}}^{+} \mathrm{T}$ cells in vitro ${ }^{97}$. These cells were termed Delta One T cells. TCR ligation and IL-15 supplementation induce the expression of NKRs such as NKp30 and NKp44. Another study showed that $\mathrm{V} \delta 1^{+}$ $\mathrm{T}$ cells derived in vitro can recognize the melanoma antigens MART1 and gp100. MART1- and gp100-reactive $\mathrm{V} \delta 1^{+} \mathrm{T}$ cells were restricted to HLA-A2. This is the first evidence of $\mathrm{MHC}$ restriction in $\gamma \delta \mathrm{T}$ cells ${ }^{24}$. This knowledge can be used to develop MHC-restricted $\gamma \delta \mathrm{T}$ cell therapies and vaccines.

Recently, $\gamma \delta \mathrm{TCR}$-based chimeric antigen receptor (CAR)-T cells were developed. Although their therapeutic efficacy remains mostly unknown, many people expect $\gamma \delta$ CAR $T$ cells to be beneficial in attenuating cytokine release syndrome and neurotoxicity ${ }^{7}$. A recent study showed that $\gamma \delta$ CAR $T$ cells are active against leukemia in vitro and in vivo. However, these cells have limited persistence $^{98}$. Because $\gamma \delta$ CAR-T cells might be effective alternatives for use against antigen-negative or MHC-low tumor cells, further development and research should be performed. In addition to the cells exhibiting direct cytotoxicity and migration ability, $\gamma \delta$ CAR-T cells developed by Capsomidis et al. ${ }^{99}$ showed the cross-presentation ability to $\mathrm{T}$ cells. To maximize the advantages of each of these $\mathrm{T}$-cell types, $\mathrm{V} \gamma 9 \mathrm{~V} \delta 2 \mathrm{TCRs}$ have been transduced into $\alpha \beta \mathrm{T}$ cells ${ }^{100}$. These cells are termed T cells engineered with defined gamma delta TCRs (TEGs). When $\gamma \delta$ TCR genes are transduced into CD4 T cells, CD4 TEG cells exhibit both cytotoxicity and helper activity that can aid in the maturation of DCs. Furthermore, because $\alpha \beta$ $\mathrm{T}$ cells express low levels of inhibitory KIRs, $\gamma \delta$ TCRmediated cytotoxicity is less inhibited ${ }^{89}$. Furthermore, a recent study has shown that natural TEGs in CNS inflammation environments display enhanced effector functions ${ }^{33}$. If these cells can be isolated or expanded, they can be beneficial for clinical use.

\section{Future perspectives}

Because $\gamma \delta \mathrm{T}$ cells constitute a minor cell population, their importance in multiple diseases has been neglected. Furthermore, the homologs in animal models and humans do not match. In particular, DETCs do not exist in humans. Because neither information about TCR ligands nor a system for expanding antigen-specific murine $\gamma \delta$ $\mathrm{T}$ cells in vitro and ex vivo are available, investigating $\gamma \delta$ $\mathrm{T}$ cells has been relatively challenging. However, recent studies have emphasized the unexpected importance of $\gamma \delta$ $\mathrm{T}$ cells, specifically in multiple tumor types. Despite the low number of $\gamma \delta \mathrm{T}$ cells during homeostasis, a large number of $\gamma \delta \mathrm{T}$ cells are recruited to tumor sites to perform effector functions. In some tumors, $\gamma \delta \mathrm{T}$ cells exhibit more antitumor activity than conventional $\mathrm{T}$ cells. For this reason, we anticipate the development of $\gamma \delta \mathrm{T}$ cell-based therapies in the future even though clinical results have been disappointing thus far. Several studies have suggested that the roles of $\gamma \delta \mathrm{T}$ cells are very complicated and context-dependent. Thus, three aspects must be determined to successfully use $\gamma \delta \mathrm{T}$ cells as therapeutic agents in the clinic. First, we need to identify cognitive murine $\gamma \delta$ TCR antigens. Although the use of several antigens, including Annexin-A2 and Skint1, has been suggested, expansion of peripheral $\gamma \delta \mathrm{T}$ cells in vitro has not been realized. As a result, it is difficult to generate ex vivo cultures or perform adoptive transfers using murine $\gamma \delta \mathrm{T}$ cells in immunocompetent mice. Transfer models of $\gamma \delta$ T cells using immunodeficient mice have obvious limitations because the effects of other immune cells on $\gamma \delta \mathrm{T}$ cells are excluded. Furthermore, tumor 
antigens that can be recognized by $\gamma \delta \mathrm{T}$ cells must be identified before $\gamma \delta \mathrm{T}$ cells can be used for therapy. Second, investigations of $\gamma \delta$ T cells in the TME using highend technology are needed. Recently, scRNA-seq, cytometry by time of flight, and spatial transcriptomics have provided insightful information at the single-cell level. Because $\gamma \delta \mathrm{T}$ cells are highly heterogeneous, it will be helpful to understand complex $\gamma \delta$ T-cell biology. Third, a systemic review of the roles of $\gamma \delta$ T cells in a case-by-case manner is needed even though the results from these studies can sometimes be stochastic and inconsistent. Upon completion of the first and second steps, we must summarize and classify the complex $\gamma \delta$ T-cell functions. Once we understand more about $\gamma \delta \mathrm{T}$ cells, they may become game-changing tools in the fight against cancer.

\section{Acknowledgements}

This work was supported by the National Research Foundation of Korea (NRF2018M3A9H3024611). Figures were created with BioRender.com.

\section{Conflict of interest}

The authors declare no competing interests.

\section{Publisher's note}

Springer Nature remains neutral with regard to jurisdictional claims in published maps and institutional affiliations.

Received: 13 December 2020 Revised: 12 January 2021 Accepted: 14 January 2021.

Published online: 12 March 2021

\section{References}

1. Chien, Y. et al. A third type of murine T-cell receptor gene. Nature 312,31-35 (1984).

2. Hayday, A. C. et al. Structure, organization, and somatic rearrangement of T cell gamma genes. Cell 40, 259-269 (1985).

3. Hayday, A. C. Gammadelta T cells and the lymphoid stress-surveillance response. Immunity 31, 184-196 (2009).

4. Willcox, C. R., Mohammed, F. \& Willcox, B. E. The distinct MHC-unrestricted immunobiology of innate-like and adaptive-like human gammadelta $T$ cell subsets-Nature's CAR-T cells. Immunol. Rev. 298, 25-46 (2020).

5. Prinz, I., Silva-Santos, B. \& Pennington, D. J. Functional development of gammadelta T cells. Eur. J. Immunol. 43, 1988-1994 (2013).

6. Ribot, J. C., Lopes, N. \& Silva-Santos, B. gammadelta T cells in tissue physiology and surveillance. Nat. Rev. Immunol. (2020). https://doi.org/10.1038/ s41577-020-00452-4.

7. Silva-Santos, B., Mensurado, S. \& Coffelt, S. B. gammadelta T cells: pleiotropic immune effectors with therapeutic potential in cancer. Nat. Rev. Cancer 19, 392-404 (2019).

8. Chien, Y. H., Meyer, C. \& Bonneville, M. gammadelta T cells: first line of defense and beyond. Annu. Rev. Immunol. 32, 121-155 (2014).

9. Gentles, A. J. et al. The prognostic landscape of genes and infiltrating immune cells across human cancers. Nat. Med. 21, 938-945 (2015).

10. Shafi, S. et al. An NKG2D-mediated human lymphoid stress surveillance response with high interindividual variation. Sci. Transl. Med. 3, 113ra124 (2011)

11. Lanier, L. L. NKG2D receptor and its ligands in host defense. Cancer Immunol. Res. 3, 575-582 (2015).

12. Girardi, M. et al. Regulation of cutaneous malignancy by gammadelta $T$ cells. Science 294, 605-609 (2001).

13. Street, S. E. et al. Innate immune surveillance of spontaneous B cell lymphomas by natural killer cells and gammadelta T cells. J. Exp. Med. 199, 879-884 (2004).
14. Liu, Z. et al. Protective immunosurveillance and therapeutic antitumor activity of gammadelta $T$ cells demonstrated in a mouse model of prostate cancer. $\rfloor$ Immunol. 180, 6044-6053 (2008).

15. He, W. et al. Naturally activated $V$ gamma 4 gamma delta $T$ cells play a protective role in tumor immunity through expression of eomesodermin. $\mathrm{J}$. Immunol. 185, 126-133 (2010).

16. Chauvin, C. et al. NKG2D controls natural reactivity of VgammagVdelta2 $T$ lymphocytes against mesenchymal glioblastoma cells. Clin. Cancer Res. 25, 7218-7228 (2019).

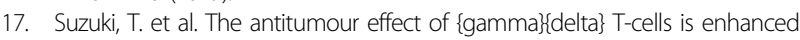
by valproic acid-induced up-regulation of NKG2D ligands. Anticancer Res. 30, 4509-4513 (2010).

18. Sun, L. L. et al. Zoledronic acid sensitizes rhabdomyosarcoma cells to cytolysis mediated by human gammadelta T cells. Oncol. Lett. 14, 5597-5604 (2017)

19. Rincon-Orozco, B. et al. Activation of $\mathrm{V}$ gamma $9 \mathrm{~V}$ delta $2 \mathrm{~T}$ cells by NKG2D. J. Immunol. 175, 2144-2151 (2005).

20. Correia, D. V., Lopes, A. \& Silva-Santos, B. Tumor cell recognition by gammadelta T lymphocytes: T-cell receptor vs. NK-cell receptors. Oncoimmunology 2, e22892 (2013)

21. Tokuyama, $H$. et al. $\vee$ gamma $9 \mathrm{~V}$ delta $2 \mathrm{~T}$ cell cytotoxicity against tumor cells is enhanced by monoclonal antibody drugs-rituximab and trastuzumab. Int. J. Cancer 122, 2526-2534 (2008).

22. Ribot, J. C., Ribeiro, S. T., Correia, D. V., Sousa, A. E. \& Silva-Santos, B. Human gammadelta thymocytes are functionally immature and differentiate into cytotoxic type 1 effector T cells upon IL-2/L-15 signaling. J. Immunol. 192, 2237-2243 (2014).

23. $\mathrm{Yi}$, Y. et al. The functional impairment of HCC-infiltrating gammadelta $T$ cells, partially mediated by regulatory T cells in a TGFbeta- and IL-10-dependent manner. J. Hepatol. 58, 977-983 (2013).

24. Benveniste, P. M. et al. Generation and molecular recognition of melanomaassociated antigen-specific human gammadelta T cells. Sci. Immunol. $\mathbf{3}$ eaav4036 (2018).

25. Sabbione, F. et al. Neutrophils suppress gammadelta T-cell function. Eur. J. Immunol. 44, 819-830 (2014)

26. Li, L. et al. Microenvironmental oxygen pressure orchestrates an anti- and pro-tumoral gammadelta $T$ cell equilibrium via tumor-derived exosomes. Oncogene 38, 2830-2843 (2019).

27. Rodrigues, $\mathrm{N}$. V. et al. Low-density lipoprotein uptake inhibits the activation and antitumor functions of human VgammagVdelta2 T cells. Cancer Immunol. Res. 6, 448-457 (2018).

28. Beck, B. H. et al. Dynamics of circulating gammadelta $T$ cell activity in an immunocompetent mouse model of high-grade glioma. PLOS ONE 10, e0122387 (2015)

29. Spidale, N. A., Frascoli, M. \& Kang, J. gammadeltaTCR-independent origin of neonatal gammadelta $T$ cells prewired for $\mathrm{IL}-17$ production. Curr. Opin. Immunol. 58, 60-67 (2019).

30. Jensen, K. D. et al. Thymic selection determines gammadelta T cell effector fate: antigen-naive cells make interleukin-17 and antigen-experienced cells make interferon gamma. Immunity 29, 90-100 (2008).

31. Turchinovich, G. \& Hayday, A. C. Skint-1 identifies a common molecular mechanism for the development of interferon-gamma-secreting versus interleukin-17-secreting gammadelta T cells. Immunity 35, 59-68 (2011).

32. Dechanet, J. et al. Major expansion of gammadelta T lymphocytes following cytomegalovirus infection in kidney allograft recipients. J. Infect. Dis. 179, 1-8 (1999).

33. Edwards, S. C. et al. A population of proinflammatory $T$ cells coexpresses alphabeta and gammadelta T cell receptors in mice and humans. J. Exp. Med. 217, e20190834 (2020).

34. Shen, L. et al. Immunization of Vgamma2Vdelta2 $T$ cells programs sustained effector memory responses that control tuberculosis in nonhuman primates. Proc. Natl Acad. Sci. USA 116, 6371-6378 (2019).

35. Rahman, M., Dastmalchi, F., Karachi, A. \& Mitchell, D. The role of CMV in glioblastoma and implications for immunotherapeutic strategies. Oncoimmunology 8, e1514921 (2019).

36. Gao, Y. et al. Gamma delta T cells provide an early source of interferon gamma in tumor immunity. J. Exp. Med. 198, 433-442 (2003).

37. Riond, J., Rodriguez, S., Nicolau, M. L., Al Saati, T. \& Gairin, J. E. In vivo major histocompatibility complex class I (MHCl) expression on MHCllow tumor cells is regulated by gammadelta T and NK cells during the early steps of tumor growth. Cancer Immun. 9, 10 (2009). 
38. Mao, C. et al. Tumor-activated TCRgammadelta(+) T cells from gastric cancer patients induce the antitumor immune response of TCRalphabeta $(+)$ T cells via their antigen-presenting cell-like effects. J. Immunol. Res. 2014, 593562 (2014).

39. Brandes, M., Willimann, K. \& Moser, B. Professional antigen-presentation function by human gammadelta T Cells. Science 309, 264-268 (2005).

40. Crawford, G. et al. Epithelial damage and tissue gammadelta T cells promote a unique tumor-protective lgE response. Nat. Immunol. 19, 859-870 (2018).

41. Medina, B. D. et al. Oncogenic kinase inhibition limits Batf3-dependent dendritic cell development and antitumor immunity. J. Exp. Med. 216 1359-1376 (2019).

42. Ness-Schwickerath, K. J., Jin, C. \& Morita, C. T. Cytokine requirements for the differentiation and expansion of $\mathrm{LL}-17 \mathrm{~A}$ - and $\mathrm{IL}-22$-producing human Vgamma2Vdelta2 T cells. J. Immunol. 184, 7268-7280 (2010).

43. Wakita, D. et al. Tumor-infiltrating IL-17-producing gammadelta T cells support the progression of tumor by promoting angiogenesis. Eur. J. Immunol. 40, 1927-1937 (2010).

44. Jin, C. et al. Commensal microbiota promote lung cancer development via gammadelta T. Cells Cell 176, 998-1013.e1016 (2019).

45. Caccamo, N. et al. Differentiation, phenotype, and function of interleukin-17producing human Vgamma9Vdelta2 T cells. Blood 118, 129-138 (2011).

46. Lo Presti, E. et al. Squamous cell tumors recruit gammadelta T cells producing either IL17 or IFNgamma depending on the tumor stage. Cancer Immunol. Res. 5, 397-407 (2017).

47. Wu, Y. et al. An innate-like Vdelta1(+) gammadelta T cell compartment in the human breast is associated with remission in triple-negative breast cancer Sci. Transl. Med. 11, eaax9364 (2019)

48. Rei, M. et al. Murine CD27(-) Vgamma6(+) gammadelta T cells producing IL17A promote ovarian cancer growth via mobilization of protumor small peritoneal macrophages. Proc. Natl Acad. Sci. USA 111, E3562-E3570 (2014).

49. Miller, J. E. et al. IL-17A modulates peritoneal macrophage recruitment and M2 polarization in endometriosis. Front. Immunol. 11, 108 (2020).

50. Coffelt, S. B. et al. IL-17-producing gammadelta T cells and neutrophils conspire to promote breast cancer metastasis. Nature 522, 345-348 (2015).

51. Zheng, Q. et al. IL-17A promotes cell migration and invasion of glioblastoma cells via activation of PI3KJAKT signalling pathway. J. Cell Mol. Med. 23, 357-369 (2019).

52. Wu, P. et al. gammadeltaT17 cells promote the accumulation and expansion of myeloid-derived suppressor cells in human colorectal cancer. Immunity 40, 785-800 (2014).

53. Meraviglia, S. et al. Distinctive features of tumor-infiltrating gammadelta $T$ lymphocytes in human colorectal cancer. Oncoimmunology 6, e1347742 (2017).

54. Khan, M. S. S. et al. Treatment of novel IL17A inhibitor in glioblastoma implementing 3rd generation co-culture cell line and patient-derived tumor model. Eur. J. Pharm. 803, 24-38 (2017).

55. Cui, X. et al. Analysis of CD137L and IL-17 expression in tumor tissue as prognostic indicators for gliblastoma. Int. J. Biol. Sci. 9, 134-141 (2013).

56. Hu, G. et al. Tumor-infiltrating CD39(+)gammadeltaTregs are novel immunosuppressive $\mathrm{T}$ cells in human colorectal cancer. Oncoimmunology $\mathbf{6}$, e1277305 (2017)

57. Casetti, R. et al. Cutting edge: TGF-beta1 and IL-15 Induce FOXP3+ gammadelta regulatory $T$ cells in the presence of antigen stimulation. J. Immunol. 183, 3574-3577 (2009).

58. Rutkowski, M. R. et al. Microbially driven TLR5-dependent signaling governs distal malignant progression through tumor-promoting inflammation. Cancer Cell 27, 27-40 (2015).

59. Daley, D. et al. gammadelta $T$ cells support pancreatic oncogenesis by restraining alphabeta T cell activation. Cell 166, 1485-1499.e1415 (2016).

60. Mao, Y. et al. A new effect of IL-4 on human gammadelta T cells: promoting regulatory Vdelta1 $\mathrm{T}$ cells via $\mathrm{LL}-10$ production and inhibiting function of Vdelta2 T cells. Cell Mol. Immunol. 13, 217-228 (2016)

61. McKenzie, D. R. et al. IL-17-producing gammadelta T cells switch migratory patterns between resting and activated states. Nat. Commun. 8, 15632 (2017).

62. Lanca, T. et al. Protective role of the inflammatory CCR2/CCL2 chemokine pathway through recruitment of type 1 cytotoxic gammadelta T lymphocytes to tumor beds. J. Immunol. 190, 6673-6680 (2013).

63. Ye, J. et al. Specific recruitment of gammadelta regulatory $T$ cells in human breast cancer. Cancer Res. 73, 6137-6148 (2013).
64. Glatzel, A. et al. Patterns of chemokine receptor expression on peripheral blood gamma delta T lymphocytes: strong expression of CCR5 is a selective feature of $V$ delta $2 \mathrm{~N}$ gamma 9 gamma delta $T$ cells. J. Immunol. 168, 4920-4929 (2002)

65. Yam, A. O. \& Chtanova, T. The ins and outs of chemokine-mediated immune cell trafficking in skin cancer. Front. Immunol. 10, 386 (2019).

66. Thomas, M. L., Badwe, R. A., Deshpande, R. K., Samant, U. C. \& Chiplunkar, S. V. Role of adhesion molecules in recruitment of Vdelta1 T cells from the peripheral blood to the tumor tissue of esophageal cancer patients. Cancer Immunol. Immunother. 50, 218-225 (2001).

67. Hayes, S. M., Li, L. \& Love, P. E. TCR signal strength influences alphabeta/ gammadelta lineage fate. Immunity 22, 583-593 (2005)

68. Tan, Y. X. et al. Inhibition of the kinase Csk in thymocytes reveals a requirement for actin remodeling in the initiation of full TCR signaling. Nat Immunol. 15, 186-194 (2014).

69. Wencker, M. et al. Innate-like T cells straddle innate and adaptive immunity by altering antigen-receptor responsiveness. Nat. Immunol. 15, 80-87 (2014).

70. Shin, S. B. et al. Abortive gammadeltaTCR rearrangements suggest ILC2s are derived from T-cell precursors. Blood Adv. 4, 5362-5372 (2020).

71. Christensen, M. V., Hogdall, C. K., Jochumsen, K. M. \& Hogdall, E. V. S. Annexin A2 and cancer: a systematic review. Int. J. Oncol. 52, 5-18 (2018).

72. Payne, K. K. et al. BTN3A1 governs antitumor responses by coordinating alphabeta and gammadelta T cells. Science 369, 942-949 (2020).

73. Willcox, B. E. \& Willcox, C. R. gammadelta TCR ligands: the quest to solve a 500-million-year-old mystery. Nat. Immunol. 20, 121-128 (2019).

74. Ribot, J. C., Debarros, A., Mancio-Silva, L., Pamplona, A. \& Silva-Santos, B. B7CD28 costimulatory signals control the survival and proliferation of murine and human gammadelta T cells via IL-2 production. J. Immunol. 189, 1202-1208 (2012).

75. Laird, R. M., Wolf, B. J., Princiotta, M. F. \& Hayes, S. M. gammadelta T cells acquire effector fates in the thymus and differentiate into cytokineproducing effectors in a Listeria model of infection independently of CD28 costimulation. PLOS ONE 8, e63178 (2013).

76. Ribot, J. C. et al. CD27 is a thymic determinant of the balance between interferon-gamma- and interleukin 17-producing gammadelta T cell subsets. Nat. Immunol. 10, 427-436 (2009).

77. DeBarros, A., Chaves-Ferreira, M., d'Orey, F., Ribot, J. C. \& Silva-Santos, B. CD70CD27 interactions provide survival and proliferative signals that regulate $T$ cell receptor-driven activation of human gammadelta peripheral blood lymphocytes. Eur. J. Immunol. 41, 195-201 (2011).

78. Pei, Y. et al. CD137 costimulation enhances the antiviral activity of Vgamma9Vdelta2-T cells against influenza virus. Signal Transduct. Target Ther. 5, 74 (2020)

79. Lee, S. J. et al. 4-1BB signal stimulates the activation, expansion, and effector functions of gammadelta T cells in mice and humans. Eur. J. Immunol. 43, 1839-1848 (2013).

80. Ribeiro, S. T., Ribot, J. C. \& Silva-Santos, B. Five layers of receptor signaling in gammadelta T-cell differentiation and activation. Front. Immunol. 6, 15 (2015).

81. Iwasaki, M. et al. Expression and function of PD-1 in human gammadelta T cells that recognize phosphoantigens. Eur. J. Immunol. 41, 345-355 (2011).

82. Shitara, S. et al. IL-7 produced by thymic epithelial cells plays a major role in the development of thymocytes and TCRgammadelta+ intraepithelial lymphocytes. J. Immunol. 190, 6173-6179 (2013).

83. Michel, M. L. et al. Interleukin 7 (IL-7) selectively promotes mouse and human IL-17-producing gammadelta cells. Proc. Natl Acad. Sci. USA 109, 17549-17554 (2012).

84. Zhao, H., Nguyen, H. \& Kang, J. Interleukin 15 controls the generation of the restricted $T$ cell receptor repertoire of gamma delta intestinal intraepithelial lymphocytes. Nat. Immunol. 6, 1263-1271 (2005).

85. Yin, Z. et al. Dominance of $\mathrm{IL}-12$ over $\mathrm{IL}-4$ in gamma delta $T$ cell differentiation leads to default production of IFN-gamma: failure to down-regulate IL-12 receptor beta 2-chain expression. J. Immunol. 164, 3056-3064 (2000).

86. Tsai, C. Y. et al. Type I IFNs and IL-18 regulate the antiviral response of primary human gammadelta $T$ cells against dendritic cells infected with Dengue virus. J. Immunol. 194, 3890-3900 (2015).

87. Riol-Blanco, L. et al. IL-23 receptor regulates unconventional IL-17-producing T cells that control bacterial infections. J. Immunol. 184, 1710-1720 (2010).

88. Patil, R. S. et al. IL17 producing gammadelta T cells induce angiogenesis and are associated with poor survival in gallbladder cancer patients. Int. J. Cancer 139, 869-881 (2016). 
89. Sebestyen, Z., Prinz, I., Dechanet-Merville, J., Silva-Santos, B. \& Kuball, J. Translating gammadelta (gammadelta) $T$ cells and their receptors into cancer cell therapies. Nat. Rev. Drug Discov. 19, 169-184 (2020).

90. Marlin, R. et al. Sensing of cell stress by human gammadelta TCR-dependent recognition of annexin A2. Proc. Natl Acad. Sci. USA 114, 3163-3168 (2017).

91. Siegers, G. M., Dutta, I., Lai, R. \& Postovit, L. M. Functional plasticity of gamma delta $T$ cells and breast tumor targets in hypoxia. Front. Immunol. 9, 1367 (2018).

92. Lamb, L. S. Jr et al. Engineered drug resistant gammadelta T cells kill glioblastoma cell lines during a chemotherapy challenge: a strategy for combining chemo- and immunotherapy. PLOS ONE 8, e51805 (2013).

93. Mattarollo, S. R., Kenna, T., Nieda, M. \& Nicol, A. J. Chemotherapy and zoledronate sensitize solid tumour cells to Vgamma9Vdelta2 T cell cytotoxicity. Cancer Immunol. Immunother. 56, 1285-1297 (2007).

94. Wang, Z. et al. Decitabine enhances Vgamma9Vdelta2 T cell-mediated cytotoxic effects on osteosarcoma cells via the NKG2DL-NKG2D axis. Front. Immunol. 9, 1239 (2018).
95. Wistuba-Hamprecht, K. et al. Proportions of blood-borne Vdelta1+ and Vdelta2+ T-cells are associated with overall survival of melanoma patients treated with ipilimumab. Eur. J. Cancer 64, 116-126 (2016).

96. Gubin, M. M. et al. High-dimensional analysis delineates myeloid and lymphoid compartment remodeling during successful immune-checkpoint cancer therapy. Cell 175, 1443 (2018).

97. Almeida, A. R. et al. Delta one T cells for immunotherapy of chronic lymphocytic leukemia: clinical-grade expansion/differentiation and preclinical proof of concept. Clin. Cancer Res. 22, 5795-5804 (2016).

98. Rozenbaum, M. et al. Gamma-delta CAR-T cells show CAR-directed and independent activity against leukemia. Front. Immunol. 11, 1347 (2020).

99. Capsomidis, A. et al. Chimeric antigen receptor-engineered human gamma delta T cells: enhanced cytotoxicity with retention of cross presentation. Mol. Ther. 26, 354-365 (2018).

100. Grunder, C. et al. gamma9 and delta2CDR3 domains regulate functional avidity of $T$ cells harboring gamma9delta2TCRs. Blood 120, 5153-5162 (2012). 